Extrême-Orient Extrême-Occident

\section{Extrême-Orient Extrême-Occident}

$41 \mid 2017$

Statuts et identités dans l'Asie prémoderne (XVII $-\mathrm{XIX}$ siècle)

\title{
Asservir pour punir : la nature pénale du statut d'esclave dans la Chine des Ming (1368-1644)
}

Enslavement as a Punishment: Considerations about the Penal Nature of Slavery in Ming China (1368-1644)

奴婢者，罪人也。從明代的法律規則來探討奴婢的身份及其根本特 性。

\section{Claude Chevaleyre}

\section{OpenEdition}

\section{Journals}

\section{Édition électronique}

URL : http://journals.openedition.org/extremeorient/708

DOI : 10.4000/extremeorient.708

ISBN : $978-2-84292-741-7$

ISSN : $2108-7105$

Éditeur

Presses universitaires de Vincennes

\section{Édition imprimée}

Date de publication : 23 novembre 2017

Pagination : 93-118

ISBN : 978-2-84292-739-4

ISSN : 0754-5010

Référence électronique

Claude Chevaleyre, «Asservir pour punir : la nature pénale du statut d'esclave dans la Chine des Ming (1368-1644) », Extrême-Orient Extrême-Occident [En ligne], 41 | 2017, mis en ligne le 23 novembre 2019 consulté le 02 janvier 2020. URL : http://journals.openedition.org/extremeorient/708 ; DOI : 10.4000/ extremeorient.708 


\title{
Asservir pour punir : la nature pénale du statut d'esclave dans la Chine des Ming (1368-1644)
}

\author{
Claude Chevaleyre
}

«Bien qu'en Chine l'esclavage ait affecté les vies de millions de personnes aux époques prémoderne et moderne, en tant que référence conceptuelle celui-ci ne fut jamais suffisamment saillant pour contribuer à définir et promouvoir une rhétorique de la « liberté » susceptible d'accompagner le mouvement vers le nationalisme de la fin du $\mathrm{XIX}^{\mathrm{e}}$ et du début du XX $\mathrm{X}^{\mathrm{e}}$ siècle. Pas plus que l'esclavage n'a occupé une place prépondérante parmi les sujets d'investigation académique. En dépit du développement des études critiques, de l'histoire culturelle, des études sur le genre et les femmes, des travaux démographiques et de bien d'autres champs d'analyse susceptibles d'aborder la question de l'esclavage, l'asservissement et la coercition en Chine sont des problèmes d'ordinaire oubliés des travaux sur la société prémoderne ${ }^{1}$. »

S'il convient de nuancer l'affirmation selon laquelle le nationalisme chinois moderne se serait construit sans référence au concept de liberté absolue faute d'avoir fait l'expérience historique d'un esclavage " ancien, stable et conceptuellement absolu », le constat dressé en 2011 par Pamela Crossley n'en demeure pas moins juste ${ }^{2}$. Malgré quelques tentatives précoces visant à inclure la Chine dans la longue histoire mondiale de l'esclavage, l'historiographie moderne n'a que très marginalement associé le thème de l'esclavage à l'histoire de la Chine (pré)moderne ${ }^{3}$. Si bien que l'usage du mot « esclavage » dans le contexte de la Chine de cette époque continue de choquer les historiens de la Chine comme les spécialistes de l'esclavage.

1. Crossley $2011: 210$.

2. Crossley 2011 : 186. Sur la place de la rhétorique de l'esclavage à la fin des Qing, cf. Will 2007 : 23-27, Sun 2012.

3. Liang 1925, Wang 1925. 
Plusieurs explications peuvent être convoquées. Pamela Crossley en souligne deux. La première relève de l'idéologie ; en l'occurrence de «l'axiome conceptuel » hérité du marxisme (toujours prégnant) d'après lequel la catégorie «esclavage » ne saurait être opératoire dans l'analyse des formes de la dépendance aux périodes « féodale » et « capitalistique ». La seconde - qui combine la première à une forme d'ethnocentrisme - procède du tropisme de l'historiographie chinoise tendant à considérer l'esclavage comme un problème d'ordre ethnique. Étranger à l'histoire de la Chine proprement dite, celui-ci pourrait néanmoins être mobilisé pour caractériser les sociétés traditionnelles de ses « minorités » (Tibétains, Mandchous et Mongols en premier lieu $)^{4}$.

À ces explications, il faut encore ajouter l'ensemble des problèmes d'ordre terminologique, méthodologique et conceptuel qui ne manquent pas de se poser dès lors que l'on mobilise le concept d' « esclavage » dans des contextes étrangers aux expériences historiques à partir desquelles celui-ci a été forgé ${ }^{5}$; en particulier lorsque dans le contexte étudié les traits distinctifs de celui-ci ne nous apparaissent pas avec une immédiate évidence ${ }^{6}$; ou lorsque le jeu des aller-retour entre les catégories propres à l'observateur (l'historien) et celles de ses sources ne semble produire que plus de confusion.

Dans le cas de la Chine impériale tardive, la catégorie que les codes des Ming (1368-1644) et des Qing (1644-1911) nomment nubi et nupu résiste singulièrement aux définitions usuelles de l'esclavage ${ }^{7}$. Ceux qui la composaient n'étaient qu'exceptionnellement des captifs ou des étrangers déracinés et déportés. Objets de droits de propriété, ils pouvaient être achetés, vendus, loués ou aliénés. Ces caractères n'étaient cependant nullement distinctifs puisqu'ils

4. Crossley $2011: 210-211$. Chaque année, les autorités chinoises célèbrent l'« abolition de l'esclavage » (ou du « servage », la terminologie étant fluctuante) au Tibet en 1959.

5. Pour Orlando Patterson, une erreur fréquente consiste à exclure de la recherche toute société dans laquelle le phénomène n'atteint pas une importance structurelle ou systémique marquée. Patterson 1982 : X.

6. Ainsi que le remarque Alain Testart, le sort de l'esclave semblant s'améliorer dans les sociétés où il existe un pouvoir central fort (Adam Smith disait « arbitraire »), l'esclavage y «prend des formes adoucies telles qu'on ne le reconnaît point » et ne semble pas assez important pour que l'on s'y intéresse. Testart $2001: 52-53$, Smith 1776 : liv. 4, chap. 7, 208.

7. L'objet du présent article n'est pas de justifier le choix de rendre cette catégorie par « esclaves ». Je n'entends pas que ce parti pris soit accepté sans réticence. S'il mérite assurément d'être débattu, il ne saurait être disqualifié sur la base de simples présupposés (parce que c'est «la Chine », parce que la période considérée est « féodale », ou parce que nous avons à faire à une forme de servitude endogène). 
se retrouvaient dans nombre de relations sociales - y compris celles relevant de la parenté (mariage, concubinage, adoption). Toujours centrale dans les définitions de l'esclavage, la notion de «propriété » peut donc difficilement être retenue comme distinctive de leur statut ${ }^{8}$. L'examen de leurs conditions de vie ne permet pas plus d'identifier un état unique qui les distinguerait des autres catégories de sujets de l'empire : la pauvreté et la violence (si souvent mises en exergue par l'historiographie) n'étaient en rien caractéristiques de la condition des nubi, parmi lesquels se trouvait d'ailleurs une minorité d'individus riches, influents et privilégiés ${ }^{9}$. Enfin, dresser l'inventaire de leurs fonctions n'est guère plus éclairant : majoritairement affectés à des tâches domestiques et de production, les sources montrent qu'en pratique ils étaient présents dans tous les domaines d'activités et qu'ils pouvaient endosser une infinie variété d'identités économiques et sociales (lesquelles dépendaient uniquement du bon vouloir de leur maître) ${ }^{10}$. En d'autres termes, ils ne constituaient pas une « classe sociale » au sens strict du terme.

Le défi méthodologique et conceptuel que nous oppose le cas des nubi ne saurait pourtant justifier le faible intérêt qu'il a suscité jusqu'à présent. Les difficultés qu'il présente invitent à la prudence autant qu'à redoubler d'efforts et à multiplier les niveaux d'analyse afin d'apprécier le phénomène dans toutes ses dimensions tout en se gardant des comparaisons superficielles et des généralisations hâtives. Dans cette perspective, je me suis attaché lors d'un précédent travail à examiner le rapport entre l'identité d'esclave et la conception de la souillure en Chine impériale tardive. Ce travail m'a permis de mettre en évidence les mécanismes par lesquels les concepts confucéens d'obligation, de réciprocité et de bienveillance contribuaient à conditionner, à perpétuer et à limiter la portée pratique de la relation maître-esclave ${ }^{11}$.

J'ai évoqué à cette occasion les limites d'une approche centrée sur le statut juridique de l'esclave, en particulier lorsque celle-ci s'appuie uniquement sur le contenu du Code des grands Ming (Da Ming lï, promulgué en 1397) et se contente d'énumérer les incapacités et discriminations qui frappaient cette catégorie de personnes. Le Code ne prenant jamais la peine de proposer une définition positive de ce qu'est un esclave ou des causes de son

8. Pour une discussion générale des attributs de l'esclavage, $c f$. Miers $2004: 1$. Sur les difficultés de sa transposition sur le droit des personnes en Chine impériale, cf. Mazumdar 2001 : 89-91.

9. Yunjian zazhi $1615: 3 / 7 \mathrm{~b}-8 \mathrm{a}$.

10. On trouvera un exemple des cohortes d'esclaves servant uniquement à affirmer le prestige de leur maître dans He 1569 : 320-321.

11. Chevaleyre $2012: 243-244$. 
déclassement, la plupart des travaux se bornent en effet à mettre en exergue le caractère discriminatoire de la loi à leur égard ${ }^{12}$. Relégués dans la catégorie sociojuridique des sujets « dégradés » (jianmin) - par opposition aux sujets « ordinaires » (shumin ou liangren, littéralement les sujets « honorables ») -, les nubi faisaient effectivement l'objet d'un traitement juridique différencié ayant pour effet de les placer en situation d'infériorité permanente vis-à-vis des sujets ordinaires et des membres de la famille de leur maître. Pourtant, énumérer une série d'incapacités et de discriminations n'a jamais contribué à définir un statut et ne saurait être suffisant en soi.

L'étude du droit des esclaves n'en demeure pas moins féconde. Pour qu'elle ait un sens dans le contexte étudié, celle-ci doit néanmoins parvenir à dépasser cette première approche. Elle doit non seulement pouvoir s'appuyer sur une compréhension de ce dont ces discriminations étaient l'expression, mais aussi tenir compte de la logique générale du droit et de l'articulation entre le Code et les institutions dont celui-ci était le garant. Pour ce faire, le présent article s'attachera à identifier les caractères distinctifs du statut d'esclave tels qu'ils ressortent du projet de société du fondateur des Ming (Zhu Yuanzhang, r. 1368-1398). Une lecture des sources normatives promulguées sous son règne (réunies dans la collection des Documents institutionnels des Augustes Ming [Huang Ming zhishu] ${ }^{13}$, combinée à un examen renouvelé du Code, nous permettra notamment de souligner l'existence d'un lien conceptuel et quasi organique entre servitude et châtiment.

Cet enracinement de l'identité d'esclave dans celle de criminel n'est certes pas une innovation des Ming (ainsi que le démontrera la dernière partie de cet article consacrée à en retracer les origines). Sa mise en évidence peut en outre sembler dépourvue de pertinence lorsqu'il s'agit d'analyser les pratiques serviles, tant celles qui se développent dès le $\mathrm{XV}^{\mathrm{e}}$ siècle paraissent en contradiction avec l'esprit de la loi et des institutions. Si l'asservissement des hommes n'est certes jamais un phénomène abstrait, l'étude des cadres normatifs et conceptuels propres à chaque contexte historique où il a été pratiqué est néanmoins susceptible d'apporter des éléments d'explication quant aux formes qu'il a pu prendre. Dans le cas de la Chine impériale tardive, la centralité de l'identité criminelle de l'esclave n'a guère attiré l'attention des historiens, si bien que l'influence qu'elle a pu avoir sur les pratiques n'a jamais été véritablement interrogée. Elle pourrait pourtant expliquer, au moins

12. Wu $1961: 76$.

13. Zhang 1579. Les textes institutionnels réunis dans cette collection étaient chargés d'une force normative (constitutionnelle, oserait-on dire) parce qu'ils émanaient du «grand ancêtre» fondateur de la dynastie. 
en partie, les formes dissimulées d'asservissement que l'on voit se développer tout au long des Ming, ainsi que la fragilité accrue de la condition d'esclave.

\section{Le silence des sources institutionnelles des Ming sur la servitude}

Mis à part le Code, qui inclut diverses provisions relatives aux esclaves, la plupart des documents normatifs du règne de Zhu Yuanzhang ne leur accordent que peu d'importance ${ }^{14}$. Les esclaves n'apparaissent pas une fois dans les Instructions ancestrales des Augustes Ming (Huang Ming zuxun [1373]) ni dans les Placards pour l'instruction du peuple (Jiaomin bangwen [1398]). Les trois séries de Grandes instructions impériales (Dagao [1385-1388]) ne les mentionnent guère qu'à titre anecdotique. Le premier code de loi des Ming - les Commandements des grands Ming (Da Ming ling [1368]) - ne les évoque pour sa part qu'à trois reprises sans fournir la moindre indication quant à leur origine ou leur rôle dans la société. Il se borne à énoncer deux principes formulés différemment dans le Code : 1. les devoirs de l'esclave envers son maître sont calqués sur ceux du fils envers son père ${ }^{15} ; 2$. les servantes appartiennent à la sphère reproductive légitime du maître et le statut servile ne se transmet pas par la mère ${ }^{16}$. Les Précieuses instructions de Ming Taizu (Ming Taizu baoxun) mentionnent plus fréquemment les esclaves, mais toujours à titre anecdotique ou pédagogique : Zhu Yuanzhang y dénonce la dégénérescence des mœurs imputable au règne des Mongols en évoquant par exemple la présence d'esclaves se pavanant somptueusement vêtus sur les marchés ${ }^{17}$; ou bien il convoque de jeunes esclaves en présence de ses fils afin de mettre ces derniers en garde contre la tentation de l'oisiveté ${ }^{18}$.

Ces quelques éléments mis à part, la rareté des mentions d'esclaves dans les sources normatives du début des Ming suggère que si la servitude existait et était reconnue par le pouvoir, le législateur ne se souciait guère de l'encadrer ou d'en définir les normes et les limites. Cela signifie aussi qu'elle n'était pas conçue comme devant occuper une place significative dans la société qui était

14. À propos des textes normatifs du début des Ming mobilisés dans le présent travail, cf. Jiang 2005 : xl-lxxxviii et Farmer 1995 : chap. 3.

15. Zhang 1579: 1/46b-47a, 50b.

16. Zhang $1579: 1 / 15 \mathrm{~b}$. Les fils nés de servantes recevaient la même part d'héritage que ceux nés des épouses légitimes.

17. Lü $1602: 2 / 28$ a.

18. Lü $1602: 2 / 14 a-b$. 
alors en train de se construire, contrairement à l'image que nous renvoie la situation de la fin de la dynastie.

Divers éléments convergents permettent néanmoins d'aller plus loin et de brosser une esquisse des fonctions dévolues à l'institution servile dans la société idéale dont le fondateur des Ming s'efforça de dessiner les contours durant une trentaine d'années. La lecture des sources normatives fait en particulier apparaître que trois principes gouvernaient l'institution servile :

1. La possession d'esclaves était tenue pour un privilège réservé aux serviteurs de l'État ;

2. Bien que ne figurant pas dans la liste des « cinq peines » régulières (wuxing), l'asservissement des hommes n'avait d'autre fonction que de châtier ${ }^{19}$;

3. Les sujets innocents devaient par conséquent en être préservés.

\section{Un privilège réservé aux serviteurs de l'empire}

Toutes les sources institutionnelles du règne de Zhu Yuanzhang démontrent qu'en théorie les fonctionnaires et « ministres méritants » (gongchen $)^{20}$ étaient les seuls récipiendaires et possesseurs légitimes d'esclaves. Elles ne font en effet état que de deux voies d'accès à la propriété d'asservis : les personnes saisies par voie judiciaire (ou de capture) pouvaient soit être affectées au service de l'administration (moguan weinu), soit être octroyées en guise de gratification à titre personnel pour service rendu à l'empire ${ }^{21}$. Dans l'esprit du législateur, la possession d'esclaves constituait donc un privilège. Celuici était réservé en priorité aux serviteurs de l'empire s'étant distingués dans l'accomplissement de leur devoir, c'est-à-dire, du temps de Zhu Yuanzhang, ses anciens compagnons d'armes, conseillers et généraux, auxquels il octroya, une fois parvenu au pouvoir, divers privilèges, dont celui de se voir allouer un petit nombre d'esclaves et, dans certains cas, de recevoir des « régiments d'esclaves » (nujun $)^{22}$.

19. Les cinq peines régulières du Code des grands Ming étaient : 1. la petite bastonnade (chi) ; 2 la grande bastonnade (zhang) ; 3 . la servitude pénale $(t u) ; 4$. le bannissement (liu); et 5. la mort (si).

20. Étaient considérés comme ministres méritants les serviteurs de l'État qui avaient « effectivement accompli une œuvre méritoire » (guoyou gongji). Zhang 1579:3/47b.

21. Autant de pratiques qui avaient cours bien avant les Ming. Shen 1929: 400.

22. Long $1887: 970$. 
Quant aux fonctionnaires, s'ils bénéficiaient manifestement aussi de ce privilège, ils ne pouvaient en jouir que dans le cadre de leurs fonctions et dans la limite de quotas, ainsi qu'en atteste un édit de 1391 relatif à la composition des suites officielles des mandarins :

Quant aux esclaves au service des familles de fonctionnaires, en se référant au système des Tang, ils ne pourront excéder le nombre de vingt pour les familles princières et nobiliaires, de douze pour les fonctionnaires du premier rang, de dix pour ceux du deuxième rang, et de huit pour ceux du troisième rang. [Les esclaves] en surnombre devront accomplir la corvée comme le restant de la population ${ }^{23}$.

À la liste des personnes jouissant du privilège de posséder des esclaves, outre les membres de l'aristocratie princière et nobiliaire, il faut encore ajouter les fonctionnaires militaires ${ }^{24}$.

À l'autre extrémité de la hiérarchie sociale, les sujets ordinaires de l'empire (y compris les lettrés n'ayant pas rang dans l'administration impériale) se voyaient interdire la possession d'esclaves ${ }^{25}$. Le Code est on ne peut plus clair à ce propos et la majorité des juristes des Ming s'accordent sur ce point :

Les gens ordinaires et les sujets dégradés se doivent fondamentalement de travailler dur et au mieux de leurs capacités. Ils ne doivent posséder ni entretenir d'esclaves. Seules les familles des ministres méritants en possèdent. Les gens ordinaires qui possèdent et entretiennent des esclaves outrepassent leur condition. [...] C'est ainsi que l'on fait la différence entre l'honorable et le vil et c'est cela qui permet de rectifier l'ordre naturel, de consolider les coutumes et de prévenir le désordre ${ }^{26}$.

La propriété d'esclaves semble donc relativement bien encadrée. Il demeurait toutefois un vide réglementaire concernant les esclaves que pouvaient posséder l'ensemble des fonctionnaires à titre privé. Du texte établissant des quotas, l'on serait tenté de déduire qu'il limitait l'usage et la possession des esclaves aux seuls fonctionnaires du troisième au premier rang à l'exclusion de tout autre.

23. 官員之家役使奴婢，稽考唐制，王公之家不過二十人，官一品不過十二人，二 品不過十人, 三品不過八人。如有多餘之數, 與民一體當差. Ming Taizu shilu： 3118 .

24. Tan $1650: 1 / 11 b$. Shen $1587: 178 / 12 b$.

25. Guan $1602: 2 / 61 \mathrm{a}$.

26. 庶民下賤, 本當服勤致力, 不得存養奴婢。惟功臣家有之。庶人而存留畜養, 是僭分矣，故杖一百，其存養男女即放從良。此別貴賤之等，是皆正倫理厚風 俗而窒亂源. Gao 1610 : 4/9b, 11a. 
Ce texte se plaçant dans une logique d'encadrement des « suites » officielles, il pourrait aussi être interprété comme ne s'appliquant qu'au versant public de la vie des fonctionnaires et non à son versant privé. Zhu Yuanzhang n'avait en effet ni explicitement interdit aux fonctionnaires des six derniers rangs de posséder des asservis, ni décrété que les fonctionnaires ne pouvaient s'en procurer sur le marché pour les employer à titre privé. À la fin de la dynastie, l'interprétation selon laquelle toute famille de fonctionnaire pouvait légitimement acquérir des esclaves (sans que soit jamais précisée la provenance de ces esclaves privés) devait donner lieu à d'interminables controverses ${ }^{27}$.

Il semble toutefois peu probable que cette interprétation ait été conforme à l'idée que s'en faisait Zhu Yuanzhang. Le privilège accordé « à la noblesse et au mérite » (selon une formule de Guan Zhidao [1536-1608] ${ }^{28}$ n' autorisait en principe pas ceux qui en jouissaient à asservir eux-mêmes des sujets de l'empire ; pas plus qu'il ne constituait un blanc-seing autorisant la possession illimitée d'esclaves. Zhu Yuanzhang prit fréquemment prétexte de la possession excessive d'esclaves pour critiquer ses ministres ou incriminer ses adversaires politiques ${ }^{29}$. Dans son esprit, le peuple « se [devait] fondamentalement de consacrer toutes ses forces à travailler dur », et non se reposer sur le travail d'autrui ${ }^{30}$. Quant aux fonctionnaires, l'empereur passa la majeure partie de ses années de règne à dénoncer leur propension à harasser le peuple. L'asservissement de sujets ordinaires pour leur seul profit faisait partie des multiples formes de prédation contre lesquelles il entendait protéger son peuple $^{31}$. Plus généralement, il œuvra à préserver la population de toute forme d'asservissement non judiciaire par des mesures de sauvegarde ad hoc et par la restriction de l'origine des esclaves aux seuls criminels.

\section{Prévenir l'asservissement du peuple}

La principale raison à la restriction de l'usage des esclaves aux seuls serviteurs de l'État était d'ordre moral. Elle procédait de la vision que Zhu

27. Guan $1602: 2 / 62 a$. Lei $1563: 123,451$.

28. Guan $1602: 2 / 61$ a. Natif de Taicang, Guan Zhidao est connu pour son implication dans les débats intellectuels et pour sa réflexion sur la crise de la fin des Ming.

29. La possession excessive d'esclave fut par exemple retenue contre Guo Ying (13351403) et Lan Yu (?-1393), parmi tant d'autres. Zhang 1739 : 3822, 3863-3866.

30. Gao $1610: 4 / 11 \mathrm{a}$.

31. Voir le cas de Li Shuqi, qui s'attira les foudres de l'empereur pour de nombreux crimes, dont l'achat de sujets ordinaires et l'emploi d'esclaves dans sa maison. Yuzhi dagao sanbian : 78b-79a. 
Yuanzhang se faisait d'une société idéale, c'est-à-dire frugale, agraire et figée. Dans ce monde immuable, le peuple travaillait pour et par lui-même. Il n'avait pas besoin de se reposer sur une main-d'œuvre servile, ainsi que le souligne un commentaire du Miroir doré du Code des grands Ming (Da Ming xingshu jinjian [fin du XVI ${ }^{\mathrm{e}}$-début du XVII ${ }^{\mathrm{e}}$ siècle]) :

En ce qui concerne la possession d'esclaves, ce sont les mots « sujets ordinaires » qui importent. Si les familles de la population ordinaire doivent travailler dur par elles-mêmes, comment pourraient-elles posséder [des esclaves] ? C'est pourquoi cela leur est interdit ${ }^{32}$.

L' « important » était donc bien le peuple ordinaire. C'est lui que le législateur entendait tenir éloigné de la propriété d'esclaves - ce qui explique sans doute l'absence de prohibition formelle concernant leur possession par les fonctionnaires de rang inférieur. Quant aux nobles et aux fonctionnaires, l'empereur restreignit leur privilège en fixant des quotas par crainte de voir une partie de la population échapper à son autorité. Il craignait en particulier qu'une fois placés sous la protection de maîtres influents et puissants ces esclaves deviennent une source de perturbation sociale ${ }^{33}$.

Mais son acharnement à vouloir limiter l'étendue de l'institution servile reposait aussi sur des considérations d'ordre fiscal, ainsi que le suggère la mention des Annales véridiques (Shilu) indiquant que les esclaves surnuméraires des ministres méritants devaient « accomplir la corvée comme le restant de la population ». Contrairement à ce que l'on peut parfois lire, Zhu Yuanzhang ne prétendit donc jamais abolir l'institution servile ${ }^{34}$. Considérant que « son peuple » devait se consacrer aux activités agraires et qu'il était de sa responsabilité supérieure de le protéger des conséquences funestes de la faim et du froid ${ }^{35}$, il s'illustra cependant par un souci constant de préserver celui-ci de l'asservissement. Ce souci prit la forme d'un arsenal législatif strict et de mesures ponctuelles. En 1372, Zhu Yuanzhang ordonna par exemple que tous les sujets de l'empire réduits en servitude par suite des désordres et de la guerre soient émancipés ${ }^{36}$ :

32. 存養奴婢者, 重在庶民二字。庶民之家當自服勤勞, 安得存養。故以禁之 D'après Jing 1993 : 139.

33. Ming Taizu shilu : 1378-1379.

34. Hucker $1975: 288$.

35. Ming Taizu shilu : 1352.

36. Long $1887: 950$. 
Naguère, en raison des grands désordres de la fin des Yuan [1279-1368], partout le peuple chercha refuge ; qui dans son pays natal, qui loin des régions agitées par les troubles. Les personnes asservies auprès de sujets ordinaires parce qu'elles étaient isolées, faibles ou trop pauvres pour survivre, seront émancipées et rendues à la population ordinaire dès réception du présent édit. Nul ne pourra garder [autrui] asservi sous la contrainte ou le forcer à s'asservir [...]. Les familles des ministres méritants et des fonctionnaires ne sont pas concernées ${ }^{37}$.

Le fondateur des Ming prit des mesures similaires à plusieurs reprises. En 1386, il ordonna aux autorités provinciales du Henan de racheter les enfants vendus ou mis en gage par suite des catastrophes naturelles. En 1396, il fit condamner les fonctionnaires provinciaux du Jiangxi pour être " restés sans rien faire » alors que la disette contraignait les plus pauvres à vendre leurs enfants $^{38}$. Cet interventionnisme n'était cependant pas propre à la politique du fondateur des Ming. L'éminent juriste de la fin des Qing, Xue Yunsheng (1820-1901), relève que de telles mesures étaient tenues pour la marque d'un gouvernement bienveillant et qu'elles se retrouvent fréquemment, bien avant les Ming, parmi les dispositions de grâce promulguées en début de règne ou en période de famine extrême :

Dans les temps anciens, il existait une hiérarchie fixe entre l'honorable et le vil. Ceux qui se vendent parce qu'ils sont affamés sont à l'origine des personnes honorables; ils ne sont ni des inférieurs par nature ni des gens condamnés par la loi. Plongés dans ces circonstances par l'infortune et les fléaux de la guerre, ils demeurent ensuite des hommes avilis pour cent générations. Quel [souverain] serait celui qui faillirait à ses devoirs [face à cela] ${ }^{39}$ ?

La récurrence de ces mesures ad hoc combinée à la mise en place d'un arsenal juridique strict témoigne cependant d'une authentique volonté de lutter contre l'asservissement des hommes. Ce point mérite d'être souligné, tant il est oblitéré par les pratiques (contraires à l'esprit des premiers temps) que l'on observe durant la seconde moitié des Ming.

37. 曩因元末大亂, 所在人民, 或歸鄉里, 或避難地方。勢孤力弱, 或貧乏不能自 存, 於庶民之家爲奴者, 詔書到日, 即放爲良。册得羁留强令爲奴 $[\cdots]$ 。功 臣及有官之家不在此限. Fu 1539 : 2/4b-5a.

38. Ming Taizu shilu : 2688, 3550-3551.

39. 古者, 良賤有定品。以䬶餓自賣者, 本良人也, 既非氣類之本卑, 又非刑律之 收坐。不幸兵荒潛身於此, 遂將為百世賤人。屍此責者, 其誰乎？Xue 1901： 599. 
Du point de vue juridique, les voies menant à l'asservissement des hommes furent considérablement restreintes par l'inscription dans le Code de deux articles de loi. Le premier s'intitule «Recueillir et receler des enfants égarés ou perdus » (shouliu mishi zinii) ${ }^{40}$. Il visait principalement le recel et les trafics d'enfants égarés (mishi) ou en fuite (zaitao) et caractérisait trois délits distincts : la dissimulation (yincang), l'appropriation (shouliu), et la vente (mai) d'enfants perdus. Il avait pour principal effet de prohiber la plupart des formes d'appropriations de personnes (mineures mais aussi adultes) par un tiers étranger à la famille proche. Si cet article ne dit rien du rapt d'enfants non perdus ou égarés, ni des formes volontaires d'asservissement, et s'il demeure silencieux sur la capacité de vendre ou d'asservir ses propres enfants, il était complété par les dispositions d'un second article consacré aux enlèvements par la force ou la ruse.

Intitulé «Enlever une personne, enlever une personne dans le but de la vendre » (lïeren lüe mairen), ce second article caractérisait les enlèvements commis non seulement par un inconnu, mais aussi par un membre de sa propre famille $^{41}$. En substance, il prohibait l'enlèvement par stratagème (shefang lïe), par recours à la séduction (youqu) ou par la supercherie commis par un étranger à la famille, y compris avec le consentement de la victime. Il proscrivait en outre le fait de vendre un cadet ou un inférieur de sa propre famille comme esclave, y compris son propre enfant, ainsi que le confirment divers commentaires. Voici celui de Gong Ju, l'auteur d'une encyclopédie du droit des Ming à la fin de cette dynastie :

Cet article ne mentionne que le fait de vendre ses (petits-)enfants comme esclaves et non comme épouses ou concubines. Pourquoi ? Parce que le père, la mère, les oncles et les frères disposent d'un pouvoir exclusif. En vendant [leur enfant] comme esclave, ils en acceptent eux-mêmes l'outrage. [Une fille] vendue comme épouse ou concubine ne devient pas héritière. Cela n'a pas à être jugé [comme lorsqu'un fils est vendu] comme héritier [adoptif] en invoquant la loi sur le fait de transférer un (petit-)fils vers un autre patronyme en qualité d'héritier. Vendre une concubine comme servante est un délit grave tandis que vendre un (petit-)enfant comme esclave l'est moins. Parce qu'un (petit-)enfant est [une personne] que l'on a soi-même engendrée, tandis qu'une concubine porte toujours un autre patronyme ${ }^{42}$.

40. Gao $1610: 4 / 11 b-15 a$.

41. Gao $1610: 18 / 53 b-61 b$.

42. Gong (fin Ming) : 6/24b. 
Ce commentaire apporte des éléments éclairants quant à la gravité, au regard de la loi, des ventes de personnes. Vendre un individu n'était pas condamnable en soi. En revanche, deux éléments pouvaient rendre la transaction illicite : l'identité du vendeur et la destination de la personne vendue. Un père et une mère jouissaient d'une prérogative naturelle à présider au devenir de leurs descendants. De ce point de vue, vendre sa propre fille comme épouse ou concubine n'avait donc rien de condamnable. Vendre son fils comme enfant adoptif ne l'était pas plus, à condition cependant que les règles de l'adoption soient strictement respectées (transfert vers une famille portant le même patronyme et pour nulle autre finalité que d'assurer la perpétuation d'un lignage sans descendance mâle) ${ }^{43}$. Vendre sa concubine était en revanche illicite. Si l'on devait établir une analogie avec le droit de propriété, l'on pourrait dire que par le mariage, les parents transféraient à l'époux des pouvoirs relevant de l'ordre de l'usus et du fructus. Ils ne lui transféraient cependant pas le droit de disposer de leur fille (un abusus, en quelque sorte, mais restreint au droit d'aliénation et dépourvu de celui de destruction), droit qu'ils conservaient à l'état latent tant qu'aucun motif légitime ne venait mettre fin au mariage. C'est pour cette raison que vendre son enfant comme esclave était moins « grave » que de vendre sa concubine : en vendant son enfant, un père faisait simplement usage de sa prérogative de parent ; en vendant sa concubine, un époux s'arrogeait un droit qui n'était pas le sien. C'est aussi pour cela que la vente d'enfants comme esclaves était fréquente et tolérée en pratique, d'autant qu'elle était perçue comme un pis-aller préférable à la mort qui attendait les plus pauvres. Néanmoins, si la loi ne contestait pas aux parents le droit de décider du sort de leurs enfants, le choix particulier de l'asservissement avait pour effet corollaire de provoquer un avilissement (jianru). Or, dégrader ou avilir une personne par la servitude relevait du seul monopole du pouvoir judiciaire avec lequel le pouvoir de décision des parents entrait de facto en concurrence. C'est pourquoi la loi l'interdisait.

Conjugués à l'interdiction faite au peuple de posséder des esclaves, les deux articles susmentionnés restreignaient considérablement les possibilités d'asservissement d'autrui. Les seuls phénomènes que les institutions du début des Ming n'encadraient pas distinctement étaient : d'une part la possibilité pour les dignitaires et fonctionnaires d'acheter et de vendre des sujets asservis ; et d'autre part l'asservissement volontaire sans intervention d'un tiers. Ce que la loi ne précisait pas non plus, c'est l'origine de ce que l'on pourrait appeler les esclaves légaux, c'est-à-dire ceux que fonctionnaires et ministres méritants étaient autorisés à posséder dans la limite de quotas. Il est cependant clair que

43. Gao $1610: 4 / 9 b$. 
seul le processus judiciaire était investi du pouvoir de créer des esclaves et que la servitude était exclusivement pensée comme un châtiment.

\section{Un monopole de l'autorité judiciaire}

L'asservissement ne figurait pas dans la liste des peines réglementaires du système pénal des Ming. Ce n'est pourtant pas autrement qu'il apparaît dans les documents institutionnels du début de la dynastie. Plus précisément, il faisait partie des mesures de saisie judiciaire (chaozha, jimo) susceptibles d'être infligées, par extension de responsabilité, aux membres de la famille d'un condamné. À la différence de la saisie des biens matériels, celle des personnes était toutefois réservée à des cas d'une gravité extrême. Zhu Yuanzhang prononça à plusieurs reprises la saisie et l'asservissement des parents de criminels condamnés à des peines capitales ${ }^{44}$. Les Commandements des grands Ming en restreignaient quant à eux l'usage aux crimes de trahison et de rébellion :

Pour ce qui est des criminels dont les biens doivent être confisqués après inventaire, à l'exception des coupables de trahison et de rébellion, seuls peuvent être saisis les biens fonciers et bâtis ainsi que le bétail ${ }^{45}$.

Le Code réservait lui aussi les peines d'asservissement (toujours en complément de la peine principale) à un nombre restreint de crimes portant atteinte à la stabilité de l'État ou à l'intégrité de la nation. Un exemple typique est celui du traitement des proches de criminels reconnus coupables de complot de rébellion et de grande sédition (moufan dani). Parmi les plus graves qui soient, ces crimes étaient non seulement passibles de la mort par démembrement des criminels eux-mêmes, mais les pères, grands-pères, fils, petits-fils, oncles et neveux, ainsi que tous ceux qui partageaient le foyer des coupables étaient également punis de mort en application du principe de coresponsabilité pénale. Tous leurs biens étaient en outre confisqués par les autorités. La loi ne prévoyait d'épargner que les garçons de moins de quatorze ans (mineurs) et les femmes (mères, filles, épouses, concubines, sœurs et brus), qui étaient asservis et affectés au service des familles de ministres méritants ${ }^{46}$. Le même principe s'appliquait aux épouses et enfants des coupables de haute

44. Par exemple : Yuzhi dagao sanbian: 40b

45. 凡犯籍沒者, 除反叛外, 其餘罪犯止沒田產杽畜. Zhang 1579: 1/46a.

46. Gao 1610 : 18/1a-4a. 
trahison (moupan) ${ }^{47}$; aux familles de fonctionnaires coupables de manœuvres partisanes et de formation de cliques politiques ${ }^{48}$; ou encore aux moines et moniales ayant établi illégalement des monastères taoïstes et bouddhiques ${ }^{49}$. L'unique cas où la loi prévoyait l'asservissement comme peine principale était celui des Mongols et Semu (Semu ren) ${ }^{50}$ coupables de s'être mariés avec leurs semblables plutôt qu'avec des Chinois ou avec les membres d'une autre ethnie $^{51}$.

En principe, l'asservissement était donc une peine rare, essentiellement prononcée en vertu du principe de coresponsabilité pénale, réservée à des groupes spécifiques et à des cas d'une extrême gravité, et présentant un fort caractère sexué. Elle était en effet applicable aux femmes (dans le cas des implantations illégales de monastères, elle ne s'appliquait qu'aux moniales), aux mineurs de moins de quatorze ans, aux plus âgés ${ }^{52}$ et à quelques groupes ethniques minoritaires, mais jamais aux adultes de sexe masculin de la population Han. Elle se voulait donc aussi, en quelque sorte, une mesure de clémence se substituant à la mort des personnes incriminées par extension de responsabilité.

Intrinsèquement, l'asservissement était conçu comme un châtiment et donc, en théorie, comme un monopole de la puissance publique. Ce constat est confirmé par les commentaires de nombreux juristes des Ming et des Qing. L'auteur du Recueil de documents importants des Ming (Ming huiyao), Long Wenbin (1824-1893), n'envisageait que deux origines possibles aux esclaves : les captifs de guerre et les membres des familles de criminels ${ }^{53}$. Pour le grand juriste Wang Kentang (1549-1613) aussi, un esclave était par essence un criminel condamné par extension de responsabilité pénale :

Les hommes et les femmes condamnés à la servitude en vertu de l'extension de responsabilité sont différents des sujets honorables innocents. [...] Les esclaves sont des personnes confisquées par les autorités. Ils ne sont pas semblables à la population inscrite sur les registres. C'est pour cela qu'ils sont différenciés des bons sujets ${ }^{54}$.

47. Gao $1610: 18 / 4 a-6 b$.

48. Gao $1610: 2 / 37 a-40 a, 41 b-42 b$.

49. Gao $1610: 4 / 7 a-9 a$.

50. Groupes musulmans d'Asie centrale ralliés aux Mongols sous les Yuan.

51. Gao $1610: 6 / 36 a-37 b$.

52. Gao $1610: 1 / 56 \mathrm{~b}$.

53. Long $1887: 970$.

54. 男女緣坐而為奴婢, 與無罪良民不同。 [ ․] 奴婢, 是沒官之人。不齒於編讯 者，故與良人有辨. Wang $1612: 20 / 15 b$. 
La distinction que fait Wang Kentang entre, d'une part, « les hommes et les femmes condamnés à la servitude en vertu de l'extension de responsabilité » et de l'autre « les sujets honorables innocents », témoigne de ce lien presque naturel entre asservissement et châtiment ; tout comme la remarque de Xue Yunsheng établissant qu'il ne pouvait y avoir d'esclaves authentiques que les criminels (voir supra). En d'autres termes, l'esclave - comme l'ensemble des «sujets dégradés » (jianmin) - se distinguait du « sujet honorable » (liangmin, liangren), en ce qu'il était marqué par les stigmates d'un crime ou d'une faute.

\section{Asservissement et coresponsabilité pénale : un lien ancien}

La conception pénale de la servitude n'est cependant pas une innovation des Ming. Plusieurs éléments témoignent de l'existence d'un lien conceptuel, quasi organique et fort ancien, entre servitude et châtiment ; à commencer par l'incessant débat sur les origines du principe de coresponsabilité pénale (yиапzиo, lianzuo). Apparu à l'époque des Han (206 av.-220 ap. J.-C.), ce débat présente la particularité de s'articuler autour d'un ensemble de sources ayant précisément trait à la question des origines de l'institution servile. Il a eu très tôt une portée juridique, puisqu'il interroge la possibilité que le droit des anciens souverains - réputé simple et magnanime (kuanjian) - ait pu s'accommoder d'un principe aussi répressif que l'extension de la faute aux parents innocents d'un criminel ${ }^{55}$. Il s'inscrit plus largement dans le mouvement philologique d'exégèse et de reconstitution des textes canoniques, dans la mesure où il touchait aussi (et même en premier lieu) à l'interprétation des Classiques.

Plus spécifiquement, ce débat pose de manière récurrente la question du sens de la phrase « [Si vous n'obéissez pas à mes commandements], je vous ferai mettre à mort [devant les esprits de la Terre] et ferai exécuter vos enfants » (Yu ze nulu ru) que Qi, souverain légendaire de la dynastie des Xia, fit retentir à l'adresse de ses troupes avant de les mener au combat ${ }^{56}$. Toute la question repose sur le sens du caractère $n u$ qui, d'exégèses anciennes en analyses étymologiques plus récentes, a été interprété alternativement comme ayant le sens de « fils », d' « esclave », de «travail forcé » ou d' « exécution » ${ }^{57}$. Qi menaçait-il ses hommes de mort s'ils ne remplissaient pas leur devoir ou

55. Shen $1929: 385$.

56. «Serment fait à Gan» (Ganshi), Classique des documents. Cf. Kong 653: 7/2b (traduit d'après Legge $1865: 155)$.

57. Long $2003: 22-23$. Kong $653: 8 / 5 b-6 a$. 
menaçait-il d'exécuter aussi leurs fils ? Menaçait-il de les exécuter et d'asservir leurs descendants ? Ou bien menaçait-il de les exécuter ou de les asservir ?

Quel que fût le sens originel de l'expression nulu dans ce passage du Classique des documents (Shangshu), et qu'elle soit ou non le témoignage le plus ancien du principe de coresponsabilité pénale dans l'histoire juridique chinoise, l'on ne peut qu'être frappé de constater que la question des origines du principe de coresponsabilité pénale renvoie systématiquement à celle de l'asservissement. Cela ne signifie certes pas qu'il existait une relation exclusive entre esclavage et coresponsabilité pénale, mais cela révèle néanmoins une conception profondément judiciaire de l'asservissement.

Cette conception de la servitude comme étant par nature un châtiment était déjà présente dans la pensée légiste, notamment chez Shang Yang (?-338 av. J.C.), le réformateur du royaume de Qin ${ }^{58}$. On en retrouve la trace dans les Rites des Zhou (Zhouli) et leurs divers commentaires, où la servitude apparaît comme la peine privilégiée dans les cas où l'on impliquait pénalement les proches d'un criminel : "Celui qui a commis un grand crime doit le payer de sa vie. Ses fils et ses filles seront quant à eux confisqués par les autorités de la souspréfecture $^{59}$. » Elle est encore illustrée à la fin du $\mathrm{III}^{\mathrm{e}}$ siècle de notre ère dans un passage de la «Biographie de Mao Jie » (tirée de la Chronique des Trois royaumes) qui s'ouvre sur une autre citation du Classique des documents:

Depuis les saints empereurs et les rois éclairés, le châtiment s'abat aussi sur les épouses et la progéniture [du criminel]. Le Livre des documents dit : «Si ceux qui sont à droite ne remplissent pas leur office et ceux qui sont à gauche ne remplissent pas le leur, je condamnerai leurs enfants avec eux.» Parmi les fonctions du ministère de la justice se trouvait celle d'affecter les fils [de criminels] au département du service pénal et les filles [aux services] des batteurs de pilon et des travailleurs de bois sec. Selon la loi des Han, les enfants et les épouses des criminels étaient saisis et asservis. Ils étaient marqués au visage. La peine de la marque, telle que prévue dans le droit des Han, existait déjà dans les anciennes institutions. De nos jours [fin du III $^{\mathrm{e}}$ siècle de notre ère] les véritables esclaves ont pour ancêtres des criminels. Bien que cent générations se soient écoulées, l'on continue de les marquer au visage et de les offrir aux fonctionnaires ${ }^{60}$.

58. Sima Qian (91 av. J.-C.) : 2230.

59. Zhouli zhushu: 956.

60. 自古聖帝明王, 罪及妻子。書云 : 左不共左, 右不共右, 予則孥翏女。司寇之 職, 男子人于罪隷, 女子人于春亭。漢律, 罪人妻子沒為奴婢, 黝面。漢法所 行黝墨之刑, 存於古典。今真奴婢祖先有罪, 雖歷百世, 猶有騌面供官. Chen Shou (fin $\mathrm{III}^{\mathrm{e}}$ siècle) : 376. 
Certes, tant les sources transmises que les textes exhumés montrent que dès les Han la possession d'esclaves n'était déjà plus un monopole de l'autorité publique et qu'il existait à cette époque un véritable marché pour le commerce des esclaves dont les opérateurs pouvaient être aussi bien publics que privés ${ }^{61}$. Néanmoins, nombre de travaux montrent aussi que si l'usage des esclaves n'était plus réservé aux seuls services administratifs, la capacité de produire des esclaves était toujours un attribut de la puissance publique et l'esclave demeurait, au moins en théorie, l'ennemi, l'étranger ou le condamné ${ }^{62}$.

L'histoire des origines et des évolutions de la conception de l'esclavage en Chine doit encore être écrite. Mais quantité d'indices laissent à penser que ce que l'on peut observer de l'esprit des lois des Ming était déjà présent longtemps auparavant: qu'il s'agisse de la conception de l'esclavage comme un châtiment ; du lien privilégié entre peine d'asservissement et principe de coresponsabilité pénale ; de l'usage de cette peine pour des crimes particulièrement graves ${ }^{63}$; du caractère fortement sexué de la servitude ${ }^{64}$; ou bien encore de l'attitude ambivalente du pouvoir impérial vis-à-vis de l'asservissement non-judiciaire et de la possession d'esclaves à titre privé, oscillant entre laisser-faire (l'asservissement étant préférable à la mort) et interventionnisme (le bon gouvernement exigeait des empereurs qu'ils luttent contre ces pratiques, autant pour des raisons fiscales que par souci de compassion) ${ }^{65}$.

\section{Esclavage et travail pénal}

La nature pénale de l'esclavage s'exprime également (peut-être de manière plus ténue) à travers la porosité persistante entre deux phénomènes que nous tenons habituellement pour distincts, à savoir le travail pénal et l'esclavage. Celle-ci apparaît dès l'époque des Qin et des Han, à propos de laquelle Robin Yates a montré que s'il existait une distinction assez nette entre les statuts de captif, de travailleur pénal et d'esclave, ceux-ci se substituaient fréquemment les uns aux autres en fonction des circonstances et des besoins ${ }^{66}$.

Aux époques plus tardives, cette porosité entre le travail pénal et la servitude est moins immédiatement perceptible. Il est cependant évident que

\footnotetext{
61. Luo 2009 : 9-10, 13-14.

62. Luo $2009: 15$. Shen $1929: 396$.

63. Wei Zheng $636: 699$.

64. Shen $1929: 84$.

65. Shen $1929: 394,402-415$.

66. Yates $2014: 212,223$.
} 
la servitude s'est perpétuée à travers les âges (avec d'importantes variations) comme une composante du travail pénal accompagnant la plupart des peines dites « d'exil ${ }^{67}$. Au début des Ming, on observe que l'asservissement est aussi une alternative à d'autres formes de travail pénal infligées par extension de la responsabilité pénale ${ }^{68}$. Mais c'est principalement dans la définition donnée de la peine réglementaire de « servitude pénale » $(t u)$ que l'on retrouve cette proximité entre la servitude et le travail forcé.

C'est à partir des Zhou du Nord (557-581) que le terme tu aurait commencé à être employé de manière standardisée pour désigner la peine réglementaire de travaux forcés (ou « servitude pénale ») telle qu'on la retrouve dans les codes pénaux à partir des Tang. Auparavant, $t u$ désignait aussi bien le travail effectué pour un service administratif (contraint ou non) que le condamné luimême ${ }^{69}$. Sous les Ming, la peine de servitude pénale (aussi nommée tuyi ou « corvée pénale ») consistait, pour ce que l'on en sait, à déporter les condamnés et à les affecter à des tâches éprouvantes pour le compte des administrations locales $^{70}$. Selon l'auteur de fiction et néanmoins fin connaisseur du système juridique Tang Hairuo (1550-1617) :

Par $t u$ sont désignées les personnes qui, ayant commis des crimes graves, se trouvent retenues et placées sous la supervision de l'administration pour effectuer des travaux de force tels que transporter du charbon, déplacer des briques, faire bouillir le sel ou fondre le fer. Elles se consacrent entièrement à des tâches pénibles pour racheter leurs crimes jusqu'au jour où, ayant effectué leur terme, elles sont relâchées ${ }^{71}$.

Si les spécialistes de l'histoire du droit chinois traduisent aujourd'hui tu par « servitude pénale » plutôt que par «travaux forcés », c'est essentiellement parce que depuis le Code des Tang cette peine a été précisément définie comme une peine d'asservissement alors que, de toute évidence, elle s'apparente plutôt à du travail forcé. Le Code des Tang commenté et expliqué (Tang lï shuyi) énonce :

67. Constant 2016.

68. C'est ce que suggère le texte de 1384 mentionné supra.

69. Wu $2008: 176$.

70. Gao $1610: 1 / 96 a$.

71. 徒者, 謂人犯罪重, 拘收在官監遣, 力役運炭、搬磚、煎鹽、炒鐵, 一專心苦 之事, 以贖其罪, 待限滿放還. Tang (fin Ming) : 10. 
[Les condamnés à] la servitude pénale sont des esclaves, parce qu'ils sont avilis par la servitude. Il est dit dans les Rites des Zhou : «Les esclaves sont des hommes que l'on a affectés au service de la servitude pénale. » Il est aussi dit : « Ils sont chargés d'accomplir des tâches et placés dans des enceintes fermées où ils sont soumis à la discipline. » [...] Tout cela est comparable à la peine dénommée $t u$. Celle-ci est donc apparue à l'époque des $\mathrm{Zhou}^{72}$.

Ce commentaire reliant la servitude pénale à l'esclavage fut par la suite régulièrement repris par les commentateurs et juristes des Ming. Wang Qi (1530-1615) le reformula de sorte à établir une continuité historique directe entre l'asservissement pénal des Zhou, les peines de travaux forcés des Han et la peine dite $t u$ établie sous les Tang puis reprise par les Ming :

[Les condamnés à] la servitude pénale sont des esclaves, parce qu'ils sont avilis par la servitude. Cela signifie que lorsque des hommes étaient confiés au service pénal, ils étaient, selon la loi, assignés à des tâches pour le compte de l'administration. Cela correspond aux [peines] de chengdan [surveillance et construction des murailles] et de chong [décorticage du riz] de l'époque des Han. Les Tang portèrent au nombre de cinq les trois degrés du système élaboré par l'empereur Wen des Sui, et les Ming les conservèrent ${ }^{73}$.

Rappelant les débats sur la signification du caractère $n u$ dans le Classique des documents, d'aucuns considèrent cependant que la corrélation entre esclavage et peine de servitude pénale ne tenait pas compte des exégèses antérieures et procèderait d'une confusion du caractère $n u$ (esclave) avec son homophone 伎, lui-même issu d'un caractère signifiant «produire un effort » ou « extraire le travail » de quelqu'un (et non l'asservir) ${ }^{74}$.

Quoi qu'il en soit, le fait que la définition de la peine désignée par tu proposée dans les commentaires du Code des Tang renvoie aux sources mobilisées pour identifier les origines du principe de coresponsabilité pénale et de l'esclavage témoigne de l'existence d'un lien pérenne entre la coresponsabilité pénale, l'asservissement pénal, le travail forcé et (ainsi que le suggère très clairement le passage cité précédemment), les notions de dégradation personnelle et d'avilissement.

72. Zhangsun $653: 4$.

73. 徒者, 奴也。蓋奴辱之。謂男子人於罪戻, 法當任以工役。即漢之城旦春是 也。唐因隋文帝制三等加為五等, 明仍之. Wang 1586 : 4029.

74. Cai $2005: 86-87$. 


\section{Conclusion}

Pour saisir les caractères définissants du statut d'esclave en Chine impériale tardive, la présente étude s'est appuyée sur une lecture des sources institutionnelles promulguées par le fondateur des Ming. Peu loquaces lorsqu'il s'agit de proposer une définition positive de l'identité d'esclave, ces documents ont donc été largement négligés par l'historiographie du fait servile en Chine. Leur examen attentif permet cependant de dépasser le discours habituel qui consiste à réduire le statut des nubi à un ensemble, plus immédiatement perceptible il est vrai, de discriminations juridiques. Ces documents font en particulier ressortir une conception résolument pénale de la nature et des fonctions de l'asservissement des hommes. Cette conception de l'esclavage n'est assurément pas caractéristique des Ming. Le détour effectué par les sources d'époques plus éloignées a révélé l'existence d'un lien étroit et fort ancien entre l'identité d'esclave et celle de criminel. L'ensemble des débats philologiques et juridiques antérieurs aux Ming démontre en effet que l'asservissement a toujours eu pour fonction essentielle de châtier, en vertu du principe d'extension de la responsabilité pénale, les membres des familles de criminels reconnus coupables de crimes portant atteinte à la stabilité de l'État. Bien que peu innovante, la politique de Zhu Yuanzhang en matière d'esclavage se caractérise néanmoins par une volonté affirmée de réhabiliter ce principe et de rompre avec les pratiques de la dynastie précédente dont il ne cesse de dénoncer la " dégénérescence ». La prolifération de l'asservissement privé à partir du XV siècle témoigne cependant de l'échec de cette entreprise. Est-ce à dire que l'étude des normes et concepts dans lesquels s'enracine l'identité d'esclave serait sans la moindre pertinence dans l'étude du fait servile en Chine impériale tardive?

Une prise en considération accrue des normes et des concepts supposés régir les pratiques serviles ouvre potentiellement la voie à une connaissance plus fine de ces pratiques. Malgré l'écart chronique (et considérable) entre l'esprit des institutions, les moyens alloués à en faire respecter la lettre, et la réalité des pratiques, l'identité criminelle de l'esclave n'a jamais cessé, jusqu'à la fin de l'ère impériale, d'être au cœur de la conception de la servitude. Nous retrouvons ce principe dans les débats sur la décadence de la société à la fin des Ming. Il demeura aussi central dans les sources du droit domestique des Ming et des Qing, puisque les règlements claniques présentent toujours l'asservissement comme la rétribution, sinon d'un crime, à tout le moins d'une faute. Il fut encore réaffirmé avec force par les défenseurs du processus abolitionniste entre 1906 et 1910. L'invariante centralité de ce principe dans la conception de l'esclavage devrait à tout le moins conduire à s'interroger sur l'influence qu'il 
a pu exercer sur les pratiques serviles - tâche que l'historiographie a à peine effleurée à ce jour. Dans une perspective plus large, rappelons également que les normes et catégories chinoises de la servitude se retrouvent dans d'autres contextes, notamment ceux du Japon et de la Corée prémodernes, où elles ont été adaptées et ont pu contribuer à produire des systèmes parfois radicalement différents. L'histoire du fait servile en Asie orientale reste à écrire et ne saurait par conséquent faire totalement abstraction d'une approche comparative fondée sur l'étude des normes de la servitude.

\section{BIBLIOGRAPHIE}

\section{Sources primaires}

CHEN Shou 陳壽 et al. (fin III ${ }^{\mathrm{e}}$ siècle). Sanguo zhi 三國志 (Chronique des Trois royaumes). Pékin, Zhonghua shuju, 1973.

FU Fengxiang 傅鳳翔(1539). Huang Ming zhaoling 皇明詔令 (Décrets et proclamations des Augustes Ming). Repr. in Siku quanshu cunmu congshu (shibu), 58 : 1-447.

GAO Ju 高舉 (1610). Ming lï jijie fuli 明律集解附例 (Code des Ming accompagné de commentaires et des lois complémentaires). Pékin, Xiuding falüguan, 1908.

GONG Ju 貢舉 (fin Ming). Da Ming longtou biandu pangxun lïfa quanshu 大明龍頭 便讀傍訓律法全書 (Encyclopédie du droit des grands Ming commentée avec lectures complémentaires en haut de page). Tōyō Bunka, Collection Niida.

GUAN Zhidao 管志道 (1602). Congxian weisu yi 從先維俗議 (Propositions pour préserver les coutumes en suivant l'exemple des anciens). Repr. in Siku quanshu cunmu congshu (zibu), vol. 88 : 186-540.

HE Liangjun 何良俊 (1569). Siyou zhai congshuo 四友齋從說 (Euvres de l'académicien du Hanlin He [Liangjun]). Pékin, Zhonghua shuju, 1997.

KONG Yingda 孔穎達 (653). Shangshu zhengyi 尚書正義 (Sens corrigé du Classique des documents). Pékin, Zhonghua shuju, 1986.

LEI Menglin 雷夢麟 (1563). Dulü suoyan 讀律鎖言 (Notes marginales pour la lecture du Code). Pékin, Falü chubanshe, 2000.

LONG Wenbin 龍紋涁 (1887). Ming huiyao 明會要 (Recueil de documents importants des Ming). Pékin, Zhonghua shuju, 1956.

LÜ Ben 吕本 et al. (1602). Ming Taizu baoxun 明太祖寶訓 (Les précieuses admonestations de l'empereur Taizu des Ming). Taipei, Academia Sinica, 1967.

Ming Taizu [gao Huangdi] shilu 明太祖高皇帝實錄 (Annales véridiques du Haut empereur Taizu des Ming [1418]). Huang Zhangjian 黄彰健 et al. (éd.). Taipei, Zhongyang yanjiuyuan lishi yuyan yanjiusuo, 1962-1968.

SHEN Jiaben 沈家本 (1929). Lidai xingfa kao 歷代刑法考 (Étude sur le droit pénal des différentes époques). Pékin, Zhonghua shuju, 1985. 
SHEN Shixing 申時行 et al. (1587). Da Ming huidian 大明會典 (Recueil des institutions des grands Ming). Repr. in Xuxiu siku quanshu, 789-792.

SimA Qian 司馬遷 (91 av. J.-C.). Shiji 史記 (Mémoires historiques). Pékin, Zhonghua shuju, 1959.

TAN Qian 談遷 (1650). Zaolin zazu 東林雜沮 (Mélanges de la forêt des jujubiers). Repr. in Хuxiu siku quanshu, 1134-1135.

TANG Hairuo 湯海若 (fin Ming). Gujin liutiao gong'an 古今律條公案 (Cas judiciaires anciens et récents mis en relation avec les articles du Code). Shanghai, Shanghai guji chubanshe, 1990.

WANG Kentang 王肯堂 (préf. 1612). Da Ming lü fuli jianshi 大明律附例箋釋 (Une explication du Code des grands Ming avec lois complémentaires). Tōyō Bunka, collection Oki.

WANG Qi 王圻 (1586). [Ming Wanli] Xu wenxian tongkao 明萬曆續文獻通考 (Suite à l'Examen général des documents institutionnels de l'ère Wanli des Ming). Repr. in Xuxiu siku quanshu, 762.

WEI Zheng 魏徵 et al. (636). Suishu 隨書 (Histoire des Sui). Pékin, Zhonghua shuju, 1973.

XUE Yunsheng 薛允升 (1901). Tang-Ming lü hebian 唐明律合編 (Édition conjointe des lois des Tang et des Ming). Pékin, Falü chubanshe, 2000.

Yuzhi dagao sanbian 御制大誥三編 (Suite aux Grands avertissements impériaux [1387]). Repr. in Хuxiu siku quanshu 862 : 307-349.

Yunjian zazhi 雲間雜誌 (Notes variées sur Songjiang [1615]). Repr. in Siku quanshu cunmu congshu (zibu) $244:$ 476-513.

ZHANG Lu 張图 (1579). Huang Ming zhishu 皇明制書 (Documents institutionnels des Augustes Ming). Repr. in Xuxiu siku quanshu 788.

ZHANG Tingyu 張廷玉 et al. (1739). Mingshi 明史 (Histoire des Ming). Pékin, Zhonghua shuju, 1974.

ZHANGSUN Wuji 長孫無忌 et al. (653). Tanglü shuyi 唐律疏議 (Code des Tang commenté et expliqué). Pékin, Zhonghua shuju, 1983.

ZHENG Xuan 鄭玄, JIA Gongyan 賈公顏 (1815). Zhouli zhushu 周禮註疏 (Les Rites des Zhou annotés et commentés). Pékin, Beijing daxue chubanshe, 1999.

\section{Sources secondaires}

CAI Shuheng 蔡樞衡 (2005). Zhongguo xingfa shi 中國刑法史 (Histoire du droit pénal chinois). Pékin, Zhongguo fazhi chubanshe.

Chevaleyre Claude (2012). « Acting as Master and Bondservant. Considerations on Status, Identities and the Nature of "Bond-servitude" in Late Ming China ». In StANZIANI, Alessandro (dir.), Labor, Coercion, and Economic Growth in Eurasia, 17th-20th Centuries. Leyde, Brill : 237-272.

CONSTANT Frédéric (2016). «Punir par l'espace : la peine d'exil dans la Chine impériale ». Extrême-Orient, Extrême-Occident, 40 : 13-38. 
Crossley Pamela Kyle (2011). «Slavery in Early Modern China ». In Eltis David et Engerman Stanley L. (dir.), The Cambridge World History of Slavery. Cambridge, Cambridge University Press, 3 : 186-213.

FARMER Edward L. (1995). Zhu Yuanzhang and Early Ming Legislation. The Reordering of Chinese Society following the Era of Mongol Rule. Leyde : Brill.

Ho Ping-ti (1962). The Ladder of Success in Imperial China. Aspects of Social Mobility, 1368-1911. New-York, Columbia University Press.

HUCKER Charles O. (1975). China's Imperial Past. An Introduction to Chinese History and Culture. Palo Alto, Stanford University Press.

JiAng Yonglin (2005). The Great Ming Code. Da Ming Lï. Seattle, University of Washington Press.

JING Junjian 經君健 (1993). Qingdai shehui de jianmin dengji 清代社會的賤民等 級 (La strate des sujets dégradés dans la société des Qing). Hangzhou, Zhejiang renmin chubanshe.

Legge James (1865). The Shoo King or The Book of Historical Documents. Repr. Taipei, SMC Publishing, 1991.

LIANG Qichao 梁啟超 (1925). «Zhongguo nuli zhidu » (Le système esclavagiste chinois). Qinghua xuebao, 2(2) : 527-553.

LONG Ansheng 龍安生 (2003). «Shangshu Ganshi bingfei lianzuo zhidu zhi yuan » 尚 書甘誓並非連坐制度之源 (Le Serment fait à Gan du Classique des documents n'est nullement à l'origine du système de corresponsabilité pénale). Shaoguan xueyuan xuebao, 24(1) : 22-25.

LUO Kaiyu 羅開玉 (2009). « Qin Han Sanguo shiqi de nuli - Yi Chengdu weili 秦漢 三國時期的奴隸一以成都為例》(Les esclaves à l'époque des Qin, des Han et des Trois royaumes. L'exemple de Chengdu). Chengdu daxue xuebao, 6 : 9-18.

MAZUMDAR Sucheta (2011). «Rights in People, Rights in Land : Concepts of Customary Property in Late Imperial China ». Extrême-Orient, Extrême-Occident, 23 : 89107.

MIERS Suzanne (2004). «Slavery : A Question of Definition ». In CAMPBELL Gwyn (dir.), The Structure of Slavery in Indian Ocean Africa and Asia. Londres, Frank Cass : 1-16.

PATTERSON Orlando (1982). Slavery and Social Death. A Comparative Study. Cambridge [Mass.], Harvard University Press.

SMITH Adam (1843) [1776]. Recherches sur la nature et les causes de la richesse des nations. Traduit par Germain Garnier. Paris, Guillaumin.

SUN Qiang 孫強 (2012). «Wan Qing guominxing pipan zhong de nuli huayu bianxi » 晚清國民性批判中的奴隸話語辨析 (Analyse de la phraséologie esclavagiste dans la critique nationaliste de la fin des Qing). Xi'an dianzi keji daxue xuebao, 22(2) : 74-80.

TESTART Alain (2001). L'Esclave, la dette et le pouvoir. Paris, Errance.

WANG Shijie 王世杰 (1925). Zhongguo nubi zhidu 中國奴婢制度 (Le système des dépendants en Chine). Pékin, Guoli Beijing daxue chubanbu.

WILL Pierre-Étienne (2007). «L'histoire n'a pas de fin ». In DELMAS-MARTY Mireille et WILl Pierre-Étienne (dir.), La Chine et la démocratie. Paris, Fayard : 7-40. 
Claude Chevaleyre

Wu Han 吳晗 (1961). Dengxia ji 燈下集 (Recueil à la lumière de la lanterne). Pékin, Sanlian shudian.

WU Wei 伍為 (2008). 《Tu zhi yiyi yanbian » 徒之意義演變 (Les évolutions de la signification de $t u$ ). Fazhi yu shehui, $6: 176$.

YATES Robin D. S. (2014). «The Changing Status of Slaves in the Qin-Han Transition». In PINES, Yuri et al. (dir.), Birth of an Empire. The State of Qin Revisited. Berkeley, University of California Press.

\section{GLOSSAIRE}

Chaozha 抄答

Chi 笞

Da Ming lï 大明律

Ganshi 甘誓

Gongchen 功臣

Guo Ying 郭英

Guoyou gongji 果有功跡

Jimo 籍沒

Jianmin 賤民

Jianru 賤辱

Kuanjian 寬簡

Lan Yu 監玉

Lianzuo 連坐

Liangren 良人 (liangmin 良民)

Li Shuqi 李涹竘

Liu 流

Lüeren lüemai ren 罟人、畧賣人

Mai 賣

Mishi 迷失

Moguan weinu 沒官為奴

Moufan dani 謀反大逆

Moupan 謀叛

$\mathrm{Nu}$ 孥 (occurrence dans le Classique des documents); 奴/㑬 (esclave); 努 (effort)

Nubi 奴婢

Nujun 奴軍

Nupu 奴僕

Qi 启

Semu ren 色目人

Shangshu 尚書 
La nature pénale du statut d'esclave dans la Chine des Ming (1368-1644)

Shefang lüe 設方罟

Shouliu mishi zinü 收留迷失子女

Shumin 庶民

$\mathrm{Si}$ 死

$\mathrm{Tu}(\mathrm{yi})$ 徒(役)

Wuxing 五刑

Yincang 隱藏

Youqu 誘取

Yu ze nulu ru 予則拏翏汝

Yuanzuo 緣坐

Zaitao 在逃

Zhang 杖

Zhu Yuanzhang 朱元璋 
